\title{
Experimental evidence of a hiding zone in a density-near-zero acoustic metamaterial
}

Cite as: J. Appl. Phys. 129, 145101 (2021); https://doi.org/10.1063/5.0042383

Submitted: 30 December 2020 . Accepted: 19 March 2021. Published Online: 08 April 2021

(iD M. Malléjac, (iD A. Merkel, (iD J. Sánchez-Dehesa, (iD J. Christensen, (iD V. Tournat, (iD V. Romero-García, and (iD) J.P. Groby

\section{COLLECTIONS}

Paper published as part of the special topic on Acoustic Metamaterials 2021
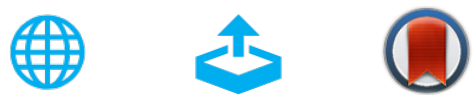

\section{HUDEN \\ Instruments for Advanced Science}

- Knowledge

- Experience,

- Expertise

Click to view our product catalogue

Contact Hiden Analytical for further details:

W www.HidenAnalytical.com E info@hiden.co.uk

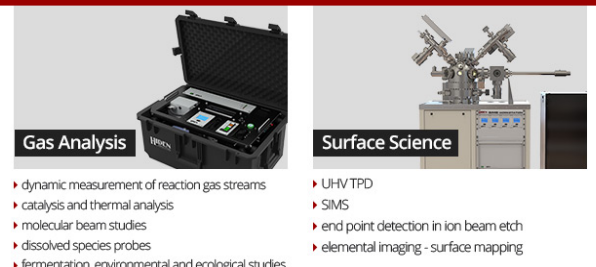

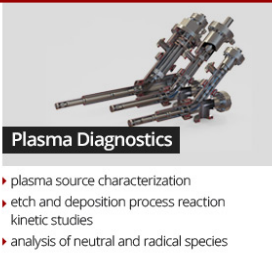

kinetic studies

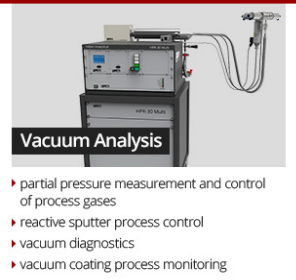

vacuum coating proces 


\title{
Experimental evidence of a hiding zone in a density-near-zero acoustic metamaterial
}

\author{
Cite as: J. Appl. Phys. 129, 145101 (2021); doi: $10.1063 / 5.0042383$ \\ Submitted: 30 December 2020 - Accepted: 19 March 2021 . \\ Published Online: 8 April 2021
}

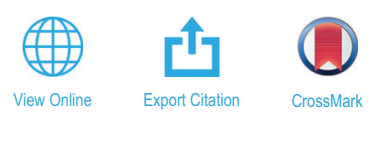

M. Malléjac, ${ }^{1, a)}$ (D) A. Merkel, ${ }^{2,3}$ (D) J. Sánchez-Dehesa, ${ }^{4}$ (D) J. Christensen, ${ }^{2}$ (iD V. Tournat, ${ }^{7}$ (iD V. Romero-García, ${ }^{1}$ and J.-P. Groby ${ }^{\top}$

\author{
AFFILIATIONS \\ ${ }^{1}$ Laboratoire d'Acoustique de I'Université du Mans (LAUM), UMR 6613, Institut d'Acoustique - Graduate School (IA-GS), CNRS, \\ Le Mans Université, Le Mans, France \\ ${ }^{2}$ Department of Physics, Universidad Carlos III de Madrid, Avenida de la Universidad 30, 28911 Leganés (Madrid), Spain \\ 3 Université de Lorraine, CNRS, IJL, F-54000 Nancy, France \\ ${ }^{4}$ Universitat Politècnica de València, Camino de Vera s/n, ES-46022 València, Spain
}

Note: This paper is part of the Special Topic on Acoustic Metamaterials 2021.

a) Author to whom correspondence should be addressed: matthieu.mallejac@univ-lemans.fr

\begin{abstract}
This paper examines the feasibility of cloaking an obstacle using Plate-type Acoustic Metamaterials (PAMs). We present two distinct strategies to cloak this obstacle, using either the near-zero-density regime of a periodic arrangement of plates or the acoustic doping phenomenon to achieve simultaneous zero-phase propagation and impedance matching. The strong limitations induced by viscothermal and viscoelastic losses that cannot be avoided in such a system are studied. A hiding zone is reported analytically, numerically, and experimentally. In contrast to cloaking, where zero-phase propagation must be accompanied by total transmission and zero reflection, the hiding configuration requires that the scattering properties of the hiding device must not be affected by the presence of the obstacle embedded in it. Contrary to cloaking, the hiding phenomenon is achievable even with a realistic PAM possessing unavoidable losses.
\end{abstract}

Published under license by AIP Publishing. https://doi.org/10.1063/5.0042383

\section{INTRODUCTION}

Cloaking is perhaps one of the most intriguing phenomena achieved through the development of metamaterials. Several strategies $^{1,2}$ have been considered to reach the quest of invisibility including both passive designs based on transformation acoustics, carpet cloaking, or extreme parameters and active designs ${ }^{3}$ based on parity-time symmetry. ${ }^{4,5}$ Transformation acoustics consists in using a coordinate transformation, ${ }^{6-10}$ as it is done in transformation optics, ${ }^{11,12}$ to deeply control the propagation of an acoustic wave. The aim is to reroute the incident wave around the obstacle to avoid any disturbance of the external field, including scattering and shadowing. A metafluid, i.e., a fluid-like material, with a controllable anisotropic density and a controllable inhomogeneity (spatially varying properties) is required to achieve that goal. ${ }^{13}$ These particular features can be obtained with a careful design of metamaterials based on either a solid inclusion-type unit cell (inertia metafluids $)^{7,14-17}$ or an interconnected network of solid bridges (pentamode metafluids). ${ }^{18-21}$ An alternative path to reach cloaking is to use topological-optimization to control wave interference in order to cancel the acoustic scattering induced by the presence of an obstacle. ${ }^{22,23}$ Another cloaking strategy, known as carpet or ground cloaking, is to cover an object placed on a reflective surface with a designed shield to make it invisible. ${ }^{24-26}$ The main limitation of this "carpet cloaking" is the large size of the device compared to the object to be masked. The development of thin metasurfaces overcomes this difficulty by using either Helmholtz resonators ${ }^{27-29}$ or membranes. ${ }^{30,31}$

An additional approach is to use extreme parameters achievable with metamaterials ${ }^{32,33}$ to cloak an obstacle from an incoming field. Density Near Zero (DNZ) metamaterials, the phase velocity of which tends toward infinity, ${ }^{34}$ are good candidates. Since the acoustic wavelength is strongly stretched in zero-index metamaterials, the presence of an obstacle inside the DNZ medium becomes almost imperceptible to an external observer. Different designs can 
be considered. Zhao et al. proposed a device based on elastic copper pieces, ${ }^{33}$ which allows, at one particular frequency, to achieve extraordinary transmission while maintaining an unchanged wave front and phase in the presence of scatterer. The DNZ condition can also result from a periodic arrangement of degenerate resonant scatterers ${ }^{35}$ or clamped elastic plates/membranes. ${ }^{36-38}$ In this latter case, strong dispersion around the resonance frequency allows to achieve zerodensity, unitary transmission, and zero-phase at three distinct frequencies in the vicinity of the bandgap edge. ${ }^{39} \mathrm{Gu}$ et al. state that cloaking is achievable in a membrane-type metamaterial and report a numerically obtained enhanced transmission through obstacles embedded in a two-dimensional square arrangement of lossless membranes. ${ }^{40}$ Nevertheless, a trade-off has to be made between the transmission magnitude and the phase shift induced by the membranes array. Yet, a perfect cloak based on this approach requires both full transmission and zero-phase propagation, which can be fulfilled by acoustic doping, i.e., the acoustic analog of photonic doping. ${ }^{41}$ The embedding of a single well-designed impurity in a membrane-type or plate-type acoustic metamaterial (PAM) can transform the DNZ medium into DCNZ (Density and Compressibility Near Zero), thus conciliating the static like field distribution and the impedance matching condition, and leading to a full non-delayed transmission in the lossless case.

In this work, we investigate the feasibility to perfectly cloak a diaphragm obstructing a cylindrical waveguide using lossless PAM in a $1 \mathrm{D}$ propagation configuration. Two distinct strategies are envisaged. The first one consists in using the diaphragm itself as an impurity to realize doping and thus cloaking, while in the second case, a Helmholtz resonator is used to dope the system leading to a full cloak of the diaphragm. As in most acoustic systems, viscothermal and viscoelastic losses cannot be neglected and are moreover known to potentially lead to the drastic annihilation of the expected phenomenon. ${ }^{42}$ In the case of doping, even if the efficiency is also limited by the losses, an enhanced transmission at the zero-phase frequency is reported in Ref. 41. The impact of losses on the effectiveness of cloaking for the two strategies considered is studied here. Furthermore, given the difficulty of achieving cloaking with a realistic PAM, emphasis is placed on the possibility of hiding a diaphragm into a PAM. The strategy is different from cloaking. Instead of trying to shield the diaphragm in the PAM to turn it invisible, we use the peculiar DNZ property to maintain the same acoustic behavior of the PAM (scattering and phase) with and without the embedded diaphragm. In this case, perfect transmission is no longer required, and a realistic (lossy) application can be considered. Finally, a hiding zone is reported experimentally in good agreement with the predictions.

\section{A. Design and modeling of the masking device}

A periodic arrangement of $N=6$ thin elastic plastic shims [Young's modulus $E_{p}=4.6\left(1+i \beta_{p}\right) \mathrm{GPa}$, density $\rho_{p}=1400 \mathrm{~kg} \mathrm{~m}^{3}$, Poisson's ratio $v_{p}=0.4$, thickness $h_{p}=102 \mu \mathrm{m}$, and loss factor $\left.\beta_{p}=0.13\right]$ equally spaced by a distance $L_{g a p}=1 \mathrm{~cm}$ and plugged into a circular waveguide of radius $R_{a}=15 \mathrm{~mm}$ and cross-sectional area $S_{a}=\pi R_{a}^{2}$ is considered here. The acoustic behavior of this PAM has been extensively studied in Ref. 39 .
The scattering parameters, i.e., the transmission coefficient $T$ and reflection coefficients $R^{+}$and $R^{-}$, of the reciprocal system are derived analytically using a transfer matrix formulation, ${ }^{43}$

$$
\begin{gathered}
R^{+}=\frac{T_{11}+T_{12} / Z_{0}-Z_{0} T_{21}-T_{22}}{T_{11}+T_{12} / Z_{0}+Z_{0} T_{21}+T_{22}}, \\
R^{-}=\frac{-T_{11}+T_{12} / Z_{0}-Z_{0} T_{21}+T_{22}}{T_{11}+T_{12} / Z_{0}+Z_{0} T_{21}+T_{22}}, \\
T=\frac{2}{T_{11}+T_{12} / Z_{0}+Z_{0} T_{21}+T_{22}},
\end{gathered}
$$

where $T_{q s}$ is the $q s$-element of the transfer matrix $\mathbf{T}$, the subscript 0 refers to the air medium, $c$ represents the speed of sound, and $Z=$ $\rho c / S_{a}$ is the characteristic impedance.

Although the single PAM is symmetrical $\left(T_{11}=T_{22}\right)$, i.e., $R^{+}=R^{-}=R$, depending on the position of the object to be concealed, the system can become asymmetrical, and both $R^{+}$and $R^{-}$ must be defined, respectively, for the reflection coefficient of an incident wave coming from the left or the right of the system.

The transfer matrix $\mathbf{T}$, linking the pressure $p$ and the flux $U$ from one side to the other of the metamaterial, takes the form

$$
\left[\begin{array}{c}
p \\
U
\end{array}\right]_{z=0}=\mathbf{T} \cdot\left[\begin{array}{c}
p \\
U
\end{array}\right]_{z=L}=\mathbf{T}_{\text {unit }^{N}} \cdot\left[\begin{array}{c}
p \\
U
\end{array}\right]_{z=L},
$$

where $\mathbf{T}_{\mathbf{u n i t}}$ is the transfer matrix of the unit cell, defined symmetrical (a clamped plate surrounded on each side by an air cavity of length $L_{\text {gap }} / 2$ ),

$$
\mathbf{T}_{\text {unit }}=\left(\mathbf{T}_{\text {cav }} \cdot\left[\begin{array}{cc}
1 & Z_{p} \\
0 & 1
\end{array}\right] \cdot \mathbf{T}_{\mathrm{cav}}\right)
$$

with

$$
Z_{p}=\frac{\Delta P}{U}=-\frac{i \omega m}{S_{a}^{2}} \frac{I_{1}\left(k_{p} R_{a}\right) J_{0}\left(k_{p} R_{a}\right)+J_{1}\left(k_{p} R_{a}\right) I_{0}\left(k_{p} R_{a}\right)}{I_{1}\left(k_{p} R_{a}\right) J_{2}\left(k_{p} R_{a}\right)-J_{1}\left(k_{p} R_{a}\right) I_{2}\left(k_{p} R_{a}\right)},
$$

the Kirchhoff-Love thin plate acoustic impedance ${ }^{37}$ with $k_{p}=\omega^{2} \sqrt{\rho_{p} h_{p} / D}$ the wave number of the flexural waves excited in the plate, $D$ is the flexural rigidity of the plate, and $m=\rho_{p} S_{a} h_{p}$ is the mass of the circular plate.

$\mathbf{T}_{\text {cav }}$ is the transfer matrix of the air cavities,

$$
\mathbf{T}_{\mathrm{cav}}=\left[\begin{array}{cc}
\cos \frac{k_{0} L_{g a p}}{2} & i Z_{0} \sin \frac{k_{0} L_{g a p}}{2} \\
\frac{i}{Z_{0}} \sin \frac{k_{0} L_{g a p}}{2} & \cos \frac{k_{0} L_{g a p}}{2}
\end{array}\right] .
$$

Note that both the wave number $k_{0}$ and the impedance $Z_{0}$ can be defined as complex valued and frequency-dependent functions to account for the viscothermal losses in the waveguide. ${ }^{44}$

In addition to the analytical calculations, $2 \mathrm{D}$ axisymmetric full-wave simulations are performed using FEM. Each plate is 
modeled as a viscoelastic medium of thickness $h$ having the aforementioned properties, fixed at the edge boundary, and fully interacting with the acoustics of the air cavities on each side. The presence of the plate induces a pressure discontinuity (fluid load on the viscoelastic structure corresponding to the pressure drop $\Delta P=p_{2}-p_{1}$, with $p_{1}$ and $p_{2}$ the acoustic pressures up and down the structure) and continuity of the flux. The system is insonified by an incident plane wave, and a plane wave radiation condition is applied at the output of the waveguide to prevent reflections.

Impedance matching, zero-density, and zero-phase propagation occur at three different frequencies, $f_{m}=423 \mathrm{~Hz}, f_{\rho=0}=414 \mathrm{~Hz}$, and $f_{\phi=0}=403 \mathrm{~Hz}$ for the lossless case. A large impedance mismatch at the zero-phase propagation frequency prevents total transmission, the magnitude of which is moreover strongly dependent on the number of unit cells, i.e., number of plates, in the system. ${ }^{39}$

Losses, mainly due to the viscoelasticity of the plate and characterized by the loss factor $\beta$, strongly alter the amplitude of the transmission at the zero-phase frequency, which drops from $\left|T_{\phi=0}\right|=0.91$ in the lossless case to $\left|T_{\phi=0}\right|=0.48$. The zero-phase frequency is shifted to $f_{\phi=0}=389 \mathrm{~Hz}$, while the maximum of transmission $\left|T_{m}\right|=0.61$ is found at $f_{m}=439 \mathrm{~Hz}$, which is the resonance frequency of the lossy plate as reported in Ref. 39.

\section{B. Design and modeling of the object to conceal}

Among the many possibilities of obstacles to conceal, the choice made in this study is a rigid diaphragm with orifice radius $R_{d}$ [sketched in Fig. 1(a)] because of the ease of controlling the scattering properties and of manufacturing. Depending on its aperture, the diaphragm switches from transparent (transmission close to 1 for large $R_{d}$ ) to opaque (reflection close to 1 for small $R_{d}$ ), thus giving one degree of freedom to adjust its influence on the scattering parameters.

The rigid diaphragm is modeled as a short waveguide of radius $R_{d}$ and length $L_{d}=2 \mathrm{~mm}$. An end correction $\Delta L_{d}=\frac{16}{3 \pi} R_{d}$, accounting for the radiation at both ends is considered and leads to an elementary transfer matrix of the form

$$
\mathbf{T}_{\mathbf{d}}=\left[\begin{array}{cc}
1 & i Z_{d} k_{d} L_{d}+i \omega \rho_{0} \Delta L_{d} \\
\frac{i}{Z_{d}} k_{d} L_{d}+i \omega\left[\kappa_{0}\right]^{-1} \Delta L_{d} & 1
\end{array}\right],
$$

in the $k_{d} L_{d} \ll 1$ approximation, with $Z_{d}$ and $k_{d}$ being the characteristic impedance and wavenumber of the orifice, and $\kappa_{0}$ being the bulk modulus of the air medium.

Figure 1(b) shows the dependence of the transmission magnitude through the diaphragm on both the frequency and $R_{d}$. The smaller the orifice is, the greater the reflection and then the lower the transmission. In addition, the transmission amplitude also decreases with frequency for the same diaphragm aperture due to the frequency-dependent viscothermal losses in the aperture. ${ }^{44}$

The shaded surface in Fig. 1(b) highlights the particular case of a diaphragm with a $R_{d}=4 \mathrm{~mm}$ orifice, the scattering parameters of which are shown in Fig. 1(c). The transmission remains high over the considered frequency range, while the magnitude of the reflection varies from 0.2 to 0.5 , thus confirming the large scattering strength of the obstacle. In addition, the presence of the diaphragm in a duct induces an increasing phase delay with frequency

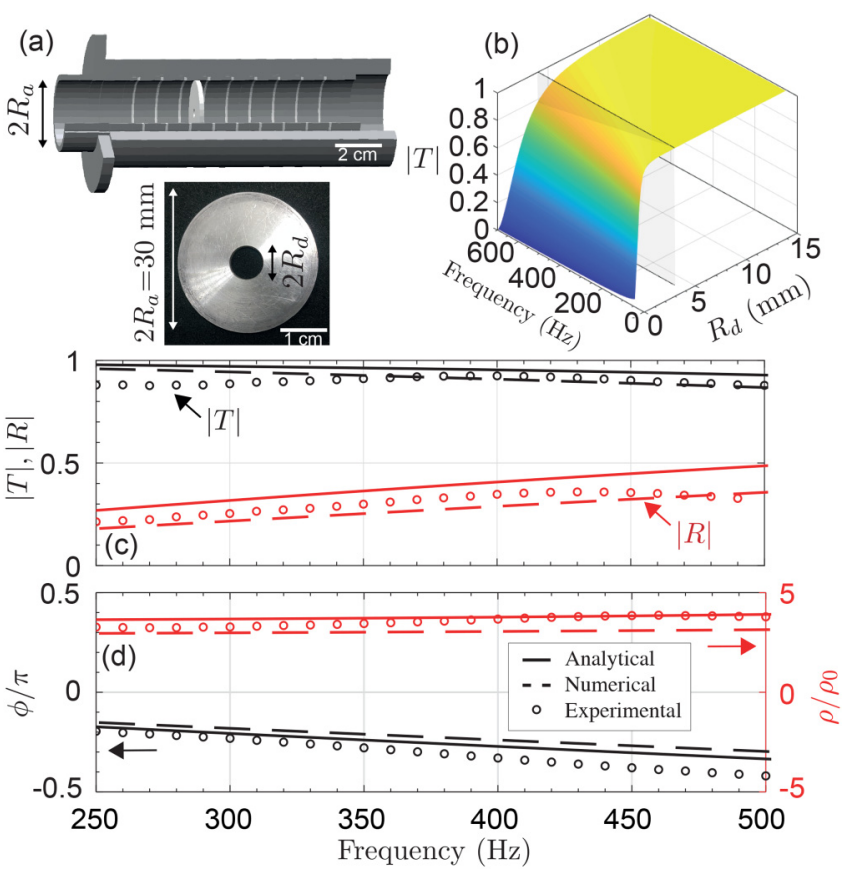

FIG. 1. Acoustic signature of a single rigid diaphragm: (a) sketch and photograph of the aluminum diaphragm, (b) 3D plot of the transmission magnitude vs frequency and the orifice radius $R_{d}$ of the diaphragm, (c) scattering parameters of a diaphragm of radius $R_{d}=4 \mathrm{~mm}$, (d) phase of the transmission coefficient (black, left axis) and normalized effective density (red, right axis). Analytical, fullwave simulation, and experimental results are given, respectively, by solid lines, dashed-lines, and symbols.

as shown by the phase of the transmission coefficient in Fig. 1(d). The designed device will then have to act on both the amplitude and phase of the scattering coefficients to enable cloaking.

\section{CLOAKING}

\section{A. Lossless system}

In the first step, we analyze the lossless case, i.e., when neither the viscoelastic losses of the plates nor the viscothermal losses in both the diaphragm orifice and the waveguide are accounted for. The diaphragm to be concealed is embedded in the middle of a 6-unit long PAM, i.e., between the 3rd and 4th plates. The two cloaking configurations under examination are optimized to find the ideal geometry to cloak the diaphragm. The efficiency of these lossless optimized systems is then tested.

\section{Cloaking configurations}

The first approach, depicted in Fig. 2(a), is to use the diaphragm itself as a dopant to match the total system impedance to the surrounding waveguide impedance at the zero-density frequency of the system. This impedance matching condition would lead to full transmission, zero reflection, and zero-phase propagation, as if the diaphragm was not present in the waveguide. 
(a)
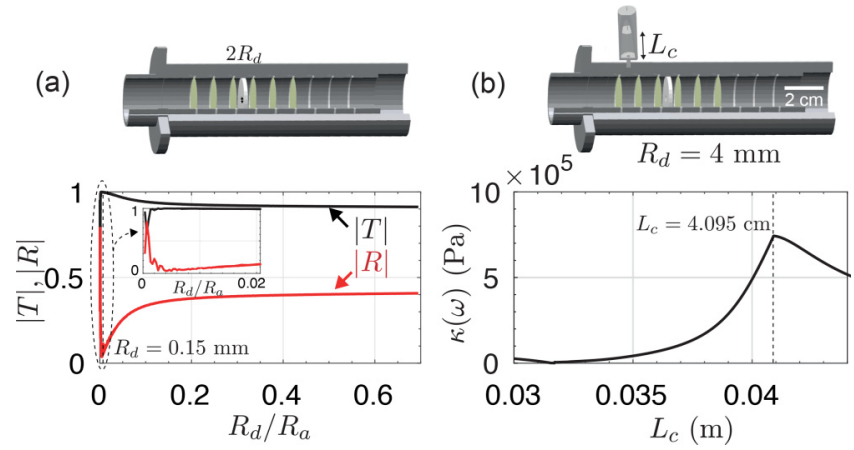

FIG. 2. Geometry optimization from analytical formulas for cloaking a diaphragm using a 6-unit long lossless PAM: (a) sketch of the configuration and influence of the diaphragm radius on the system's scattering parameters, (b) sketch of the configuration with an external dopant and optimal geometry for cloaking by doping a 6-unit long lossless PAM with a Helmholtz resonator. Evolution of the effective bulk modulus with the cavity length of the Helmholtz resonator.

The total transfer matrix characterizing the whole symmetrical system reads as

$$
\left[\begin{array}{c}
p \\
U
\end{array}\right]_{0}=\left(\mathbf{T}_{\text {unit }}\right)^{3} \cdot \mathbf{T}_{\mathbf{d}} \cdot\left(\mathbf{T}_{\text {unit }}\right)^{3}\left[\begin{array}{c}
p \\
U
\end{array}\right]_{L}
$$

The variation of the diaphragm geometry provides the optimal design for achieving cloaking. The lossless scattering magnitudes of the total system, that is, the PAM with the embedded diaphragm, are calculated analytically for different orifice radii $R_{d}$ as shown in Fig. 2(a). A perfect transmission with zero reflection is found for the optimal radius $R_{d}=0.15 \mathrm{~mm}$.

In the second configuration, an additional dopant consisting in a cylindrical Helmholtz resonator is used to dope the whole system, so as to enable cloaking. The doping condition is reached when the effective density and the effective compressibility are simultaneously equal to zero. ${ }^{41,45,46}$ A Helmholtz resonator (cavity length $L_{c}$, cavity radius $R_{c}=1 \mathrm{~cm}$, neck length $L_{n}=2 \mathrm{~cm}$, and neck radius $R_{n}=2 \mathrm{~mm}$ ) is mounted on the main waveguide, between the first two plates.

The total transfer matrix of the asymmetrical system can then be defined as follows:

$$
\left[\begin{array}{c}
p \\
U
\end{array}\right]_{0}=\mathbf{T}_{\text {unit }} \cdot \mathbf{T}_{\mathrm{HR}} \cdot\left(\mathbf{T}_{\text {unit }}\right)^{2} \cdot \mathbf{T}_{\mathbf{d}} \cdot\left(\mathbf{T}_{\text {unit }}\right)^{3}\left[\begin{array}{c}
p \\
U
\end{array}\right]_{L}
$$

The cloaking condition is found in Fig. 2(b) by varying the length of the Helmholtz resonator cavity and looking for a maximal bulk modulus, i.e., almost zero compressibility. A maximum is found for a length $L_{c}=4.1 \mathrm{~cm}$. With this particular geometry, an impedance matching condition coupled with zero-phase propagation could be achieved.

\section{Cloaking efficiency}

An analytical (solid line) and a FEM numerical study (dashed line) of the scattering parameters (magnitude and phase) and of the effective density of the two cloaking configurations are performed and presented in Figs. 3(a) and 3(b).

The numerical simulation of the self-doped configuration described in Sec. I A is performed using a 2D axisymmetric fullwave model. The doped configuration, with an additional Helmholtz resonator, requires on the contrary a $3 \mathrm{D}$ model reproducing the cylindrical impedance tube of radius $R_{a}=15 \mathrm{~mm}$ used in the experimental setup. The system is insonified by a plane incident wave propagating from left to right. A plane wave radiation condition is applied at both ends of the waveguide to avoid spurious reflections. The plates are modeled as $h_{p}$-thick viscoelastic media having the above-mentioned mechanical properties $E_{p}$, $\rho_{p}, v_{p}$. The rigid diaphragm is modeled as a $L_{d}=2 \mathrm{~mm}$-long cylindrical duct of radius $R_{d}$, located at the bounds between the 3rd and 4th unit cells of the PAM. The Helmholtz resonator, mounted on the waveguide boundary between the 1st and 2nd plates, is modeled as two air-filled cylindrical waveguides of radii $R_{n}$ and $R_{c}$ and lengths $L_{n}$ and $L_{c}$, respectively. The reflection and transmission coefficients are recovered from a 4-microphone measurement consisting of two pairs of microphones upstream and downstream of the PAM.

The two lossless configurations-self-doped diaphragm [Fig. 3(a)] and doped with a Helmholtz resonator [Fig. 3(b)]-lead to cloaking. In the first configuration, the full transmission (black color) and zero reflection (red color) observed over a very narrow frequency range in Fig. 3(a-2) result from the high impedance of the diaphragm (due to its narrow aperture). The resulting transparency is also accompanied by zero effective density (red color) and zero-phase propagation [Fig. 3(a-3)] at $f_{\phi_{t}=0}=88.3 \mathrm{~Hz}$.

Doping occurs as expected in the second configuration, when the designed Helmholtz resonator is mounted on the system. As a result, impedance matching $(|T| \approx 1,|R| \approx 0)$ [Fig. 3(b-2)] and zero-phase propagation [Fig. 3(b-3)] are also achieved at $f_{\phi_{t}=0}=403 \mathrm{~Hz}$.

The pressure field is constant (quasi-static distribution) along the system in the "Helmholtz doped configuration." In contrast, the pressure varies within the metamaterial in the "selfdoped configuration" (when the diaphragm itself enables cloaking). That change in the pressure field is, however, imperceptible to an outside observer.

Although the presence of the diaphragm becomes imperceptible in terms of scattering magnitude, both configurations do not reproduce the correct apparent phase induced by wave propagation. Indeed, measuring the phase advance of a wave propagating over a length $L=6 \mathrm{~cm}$ in an air-filled waveguide or through the proposed designs (PAM + diaphragm + potentially an additional dopant) leads to different results. The current configurations give a non-delayed propagation, that is, $\Delta \phi \approx 0$, while the phase shift to be reproduced with the device should be $\Delta \phi=k_{0} L=$ $2 \pi f_{\phi_{t}=0} / c_{0}$ (with $f_{\phi_{t}=0}=88.3 \mathrm{~Hz}$ or $f_{\phi_{t}=0}=403 \mathrm{~Hz}$ depending on the configuration) to account for the propagation. The realization of a full cloak then requires the addition of another constraint. As the whole system must be undetectable, both in terms of scattering and phase, the total length $L$ of the PAM must be chosen so that propagation over a $L$-thick slab of filling material results in a phase advance of $\Delta \phi=m 2 \pi$, with $m$ an integer. Consequently, the length of the PAM must be 

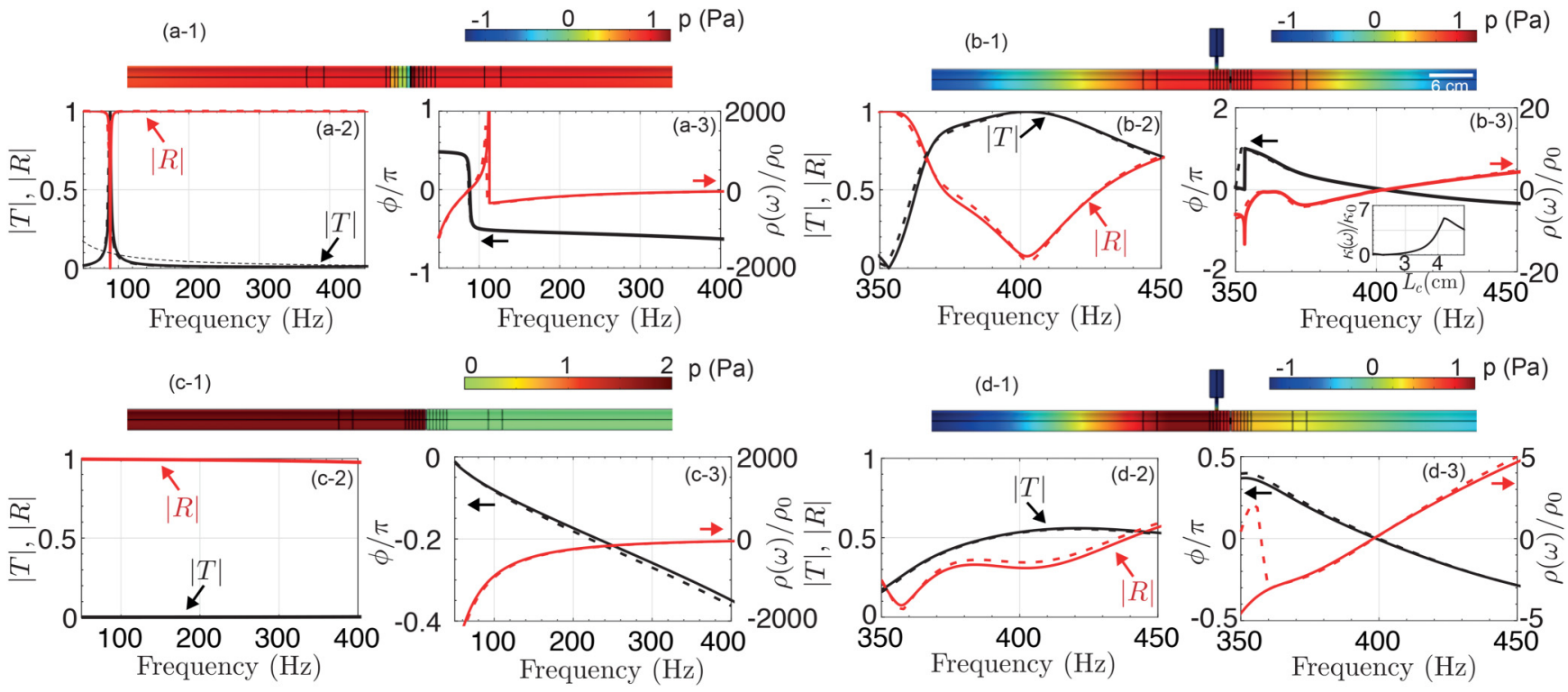

FIG. 3. (a) and (c) Cloaking of a $R_{d}=0.15 \mathrm{~mm}$ diaphragm in a conventional 6 PAM: lossless (a) and lossy (c) configurations. (b) and (d) Cloaking of a $R_{d}=4 \mathrm{~mm}$ diaphragm in a 6 PAM doped with a Helmholtz resonator $\left(R_{n}=2 \mathrm{~mm}, L_{n}=2 \mathrm{~cm}, R_{c}=1 \mathrm{~cm}\right.$, and $\left.L_{c}=40.95 \mathrm{~cm}\right)$ : lossless (b) and lossy (d) configurations. (1) Pressure field from the full-wave simulation. (2) Transmission coefficient magnitude (black) and reflection coefficient magnitude (red). (3) Phase of the transmission coefficient (black, left axis) and normalized effective density (red, right axis). The solid line represents the analytical results, and the dashed line the full-wave simulation ones.

$$
L_{o p t}=2 m \pi \frac{c_{0}}{\omega_{\phi_{t}=0}}
$$

that is, $L_{\text {opt }}=3.88 \mathrm{~m}$ for the first configuration and $L_{\text {opt }}=85.11 \mathrm{~cm}$ for the second. The overall dimensions of the cloaking device are, therefore, no longer subwavelength.

\section{B. Lossy system}

While cloaking seems to occur in lossless PAMs, we now consider the case of a realistic system, with the impact of losses, the role of which has been previously shown to be crucial in zero-index metamaterials. ${ }^{39,41,47}$

Figures 3(c) and 3(d) show the effect of total losses (viscoelasticity of the plates and viscothermal losses) on the effectiveness of the two cloaking strategies. The viscothermal losses are accounted for by the complex, frequency-dependent wave number and impedance ${ }^{44}$ in the air-filled cavities (waveguide of radius $R_{a}$, diaphragm of radius $R_{d}$, and neck of radius $R_{n}$ and cavities of radius $R_{c}$ of the Helmholtz resonator).

In the first configuration shown in Fig. 3(c), viscothermal losses in the small aperture turn the diaphragm totally opaque. The incident wave is totally reflected, as if a rigid wall is encountered. In addition, the phase of the transmission coefficient no longer passes through zero. Considering a realistic diaphragm with such an orifice then totally annihilates the cloaking effect.

In the second configuration, the viscoelasticy of the plates limits the doping efficiency as already observed in Ref. 41. As a result, the presence of the system in the waveguide induces a scattering (non-zero reflection). Losses thus also prevent cloaking in the second configuration.

\section{Feasibility of cloaking}

To summarize on the feasibility of cloaking, despite claims in the literature on the subject, this work shows that realistic applications of cloaking with membranes or plate-type metamaterials are very limited. First of all, the presence of losses in the system prevents total transparency, i.e., perfect transmission. Moreover, although the stretching of the effective wavelength produces a static-like field distribution, making the obstacle imperceptible in terms of apparent phase to an outside observer, full cloaking requires, on the contrary, reproducing the phase of an air-filled waveguide of the same length, as if the object to be cloaked and the occultation device were both absent. Full cloaking, therefore, requires specific lengths of the PAM, which are for low frequencies very large, i.e., no longer sub-wavelength.

\section{HIDING}

The goal here is to use the stretch of the effective wavelength in the metamaterial to hide the diaphragm (or any other obstacle) inside, without disturbing the acoustic scattering of the PAM itself, i.e., by maintaining a constant magnitude of reflection and transmission at the zero-phase frequency $f_{\phi=0}$.

\section{A. Centered diaphragm}

The phase shift induced by the presence of the diaphragm in a waveguide [observable in Fig. 1(d)] can be inhibited by the 
presence of a PAM in the DNZ regime. Figure 4 shows a comparison of the acoustic response (scattering parameters and effective density) of a conventional 6-unit long PAM [Figs. 4(a)-4(c)] and a 6-unit long PAM with an embedded diaphragm [Fig. 4(d)], the orifice of which is either $R_{d}=4 \mathrm{~mm}$ [Figs. 4(e) and 4(f)] or $R_{d}=1 \mathrm{~mm}$ [Figs. 4 (h)-4(i)]. In both cases, the presence of a PAM provides both zero-phase and zero-density to the whole system as evidenced in Figs. 4(f) and 4(i). A twofold procedure, including 2D axisymmetric full-wave simulations (dashed line) and experimental measurements (circle symbols), is followed to validate the TMM-based analytical predictions. A very good agreement between the numerical results, the analytical calculations, and the measurements is found. The weak discrepancies on the reflection coefficient are attributed to a remaining variability on the unit cells, due to either the clamping condition or the intrinsic properties of the plates as previously observed.

The presence of the DNZ metamaterial makes it possible to lower and even to cancel the scattering effect induced by the diaphragm. The total system (metamaterial and diaphragm) indeed achieves the non-delayed propagation property. The zero-phase frequency of the whole system $f_{\phi_{t}=0}$ depends directly on the impedance of the obstacle, related here to the open area ratio $R_{d} / R_{a}$. As shown in Fig. 4(g), the smaller the orifice radius, and, therefore, the larger the impedance of the diaphragm, the lower the zero-phase frequency of the system. The zero-phase frequency shifts from $f_{\phi=0}=390 \mathrm{~Hz}$ for the unique PAM to $f_{\phi_{t}=0}=373 \mathrm{~Hz}$ for the system with a diaphragm of radius $R_{d}=4 \mathrm{~mm}$ (green marker), and to $f_{\phi_{t}=0}=303 \mathrm{~Hz}$ for the diaphragm of $R_{d}=1 \mathrm{~mm}$ (orange marker).

At the zero-phase frequency of the system with a $R_{d}=4 \mathrm{~mm}$ diaphragm, $f_{\phi_{t}=0}=373 \mathrm{~Hz}$, the amplitudes of the measured (respectively analytical and numerical) transmission and reflection coefficients are 0.45 (respectively, 0.47) and 0.75 (respectively, 0.57) and are equal (approximately for experimental data) to the ones of the metamaterial alone at its zero-phase frequency $f_{\phi=0}$, respectively, 0.42 (respectively, 0.47) and 0.79 (respectively, 0.57). For the $1 \mathrm{~mm}$ diaphragm, the scattering parameters of the total system and of the metamaterial alone at their respective zero-phase frequencies $f_{\phi_{t}=0}$ and $f_{\phi=0}$ are not exactly the same with a transmission of 0.39 (respectively, 0.38) and a reflection of 0.59. (respectively, 0.62). The efficiency of the hiding phenomenon is, therefore, restricted when the impedance of the obstacle is excessively large. A trade-off needs to be found between the diaphragm scattering strength related to its acoustic impedance, i.e., its opening area ratio, and the efficiency of the PAM to hide the scatterer, as investigated in Fig. 5. (a)

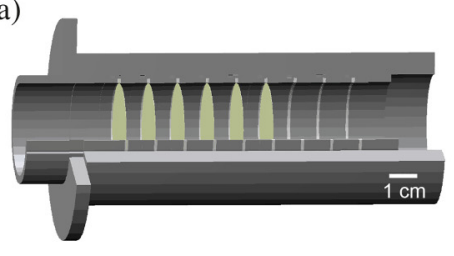

(d)
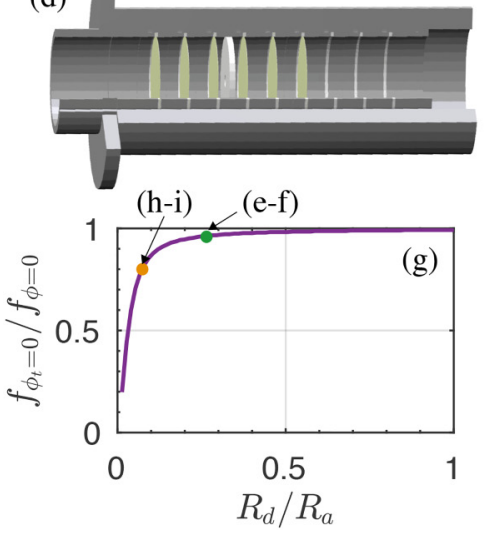
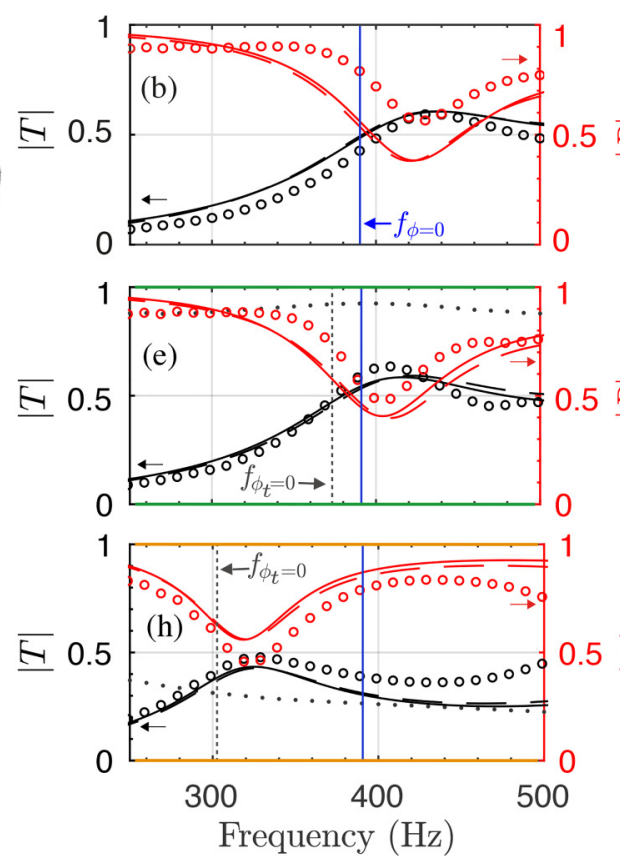
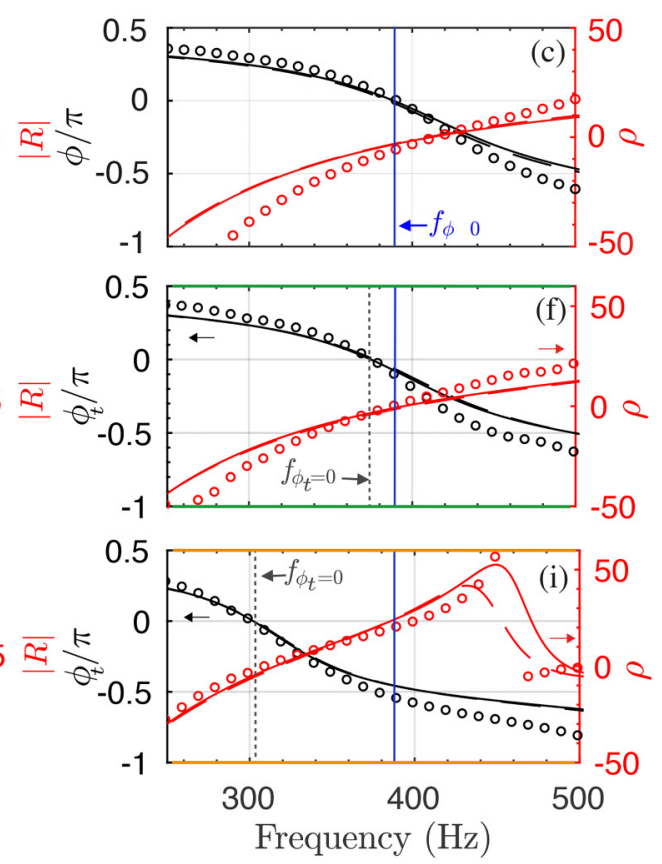

FIG. 4. Hiding of a centered embedded diaphragm. (a)-(c) 6-unit long PAM behavior $\left(h_{p}=102 \mu \mathrm{m}, L_{\text {unit }}=1 \mathrm{~cm}\right)$ : (a) sketch, (b) transmission coefficient magnitude (black, left label), reflection coefficient magnitude (red, right label), and (c) phase of the transmission coefficient (black, left label), real part of the effective dynamic mass density (red, right label). (d)-(i) 6-unit long PAM behavior with embedded $L_{d}=2 \mathrm{~mm}$ thick annular diaphragm with an aperture of radius $R_{d}$ : (d) sketch, (g) relative frequency shift [ratio of the zero-phase frequency of the transmission coefficient for the total system (diaphragm embedded into the metamaterial) to the one of the metamaterials alone] vs the diaphragm to the waveguide radii ratio (TMM). (e) and (h) transmission coefficient magnitude (black, left label) and reflection coefficient magnitude (red, right label) of the total system, metamaterial with an embedded diaphragm of aperture $R_{d}=4 \mathrm{~mm}$ and $R_{d}=1 \mathrm{~mm}$, respectively. (f) and (i) the phase of the transmission coefficient (black color) and real part of the effective dynamic mass density (red color) of the total system for $R_{d}=4 \mathrm{~mm}$ and $R_{d}=1 \mathrm{~mm}$, respectively. Black dashed curves in Figs. (e) and (h) represent the transmission of the $R_{d}=4 \mathrm{~mm}$ and $R_{d}=1 \mathrm{~mm}$ diaphragms alone. 

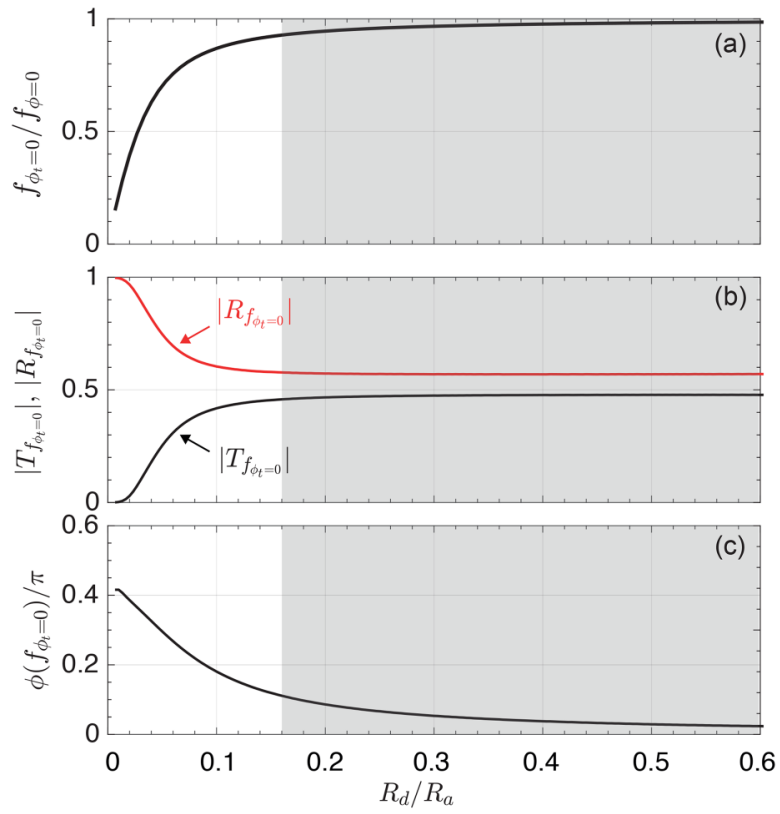

FIG. 5. Influence of the diaphragm aperture ratio on its scattering parameters (analytically calculated). (a) Variation of the zero-phase frequency. (b) Evolution of the amplitude of the scattering parameters: transmission magnitude (black, left axis) and reflection magnitude (red, right axis) at the zero-phase frequency of the total system $f_{\phi_{t}=0}$. (c) The phase of the transmission coefficient of the metamaterial alone at the zero-phase frequency of the global system $f_{\phi_{t}=0}$ for the different diaphragm radii. The shaded area shows the aperture range for which the amplitude of the scattering parameters is independent of the orifice radius.
Figure 5(b) shows that the amplitude of the scattering parameters at $f_{\phi_{t}=0}$ remains constant and equal to the ones of the metamaterial alone at $f_{\phi=0}$ as long as the ratio orifice/total cross section remains larger than $16 \%$ (gray mapped surface). Due to the slight change of the zero-phase frequency in that range, a small phase shift is noticeable for the PAM alone at the zero-phase frequency of the whole system $f_{\phi_{t}=0}$ in Fig. 5(c). Beyond that $16 \%$ ratio, the impedance of the diaphragm becomes too large. The zero-phase frequency of the whole system is consequently significantly down shifted. The $16 \%$ cross-sectional area ratio corresponds to a variation of $f_{\phi_{t}=0}$ of $10 \%$ with respect to the zero-phase frequency of the metamaterial alone $f_{\phi=0}$. Due to this frequency shift, the DNZ strength of the PAM is no longer sufficient to allow a proper hiding phenomenon. The system behaves like two distinct DNZ media separated by an obstacle of a given impedance. The scattering amplitude then varies drastically with the variation of $f_{\phi_{t}=0}$.

However, it is interesting to note that the hiding of a diaphragm (or any other obstacle) is possible with a lossy DNZ metamaterial, as soon as the impedance of the object to be hidden is well controlled. In this periodic arrangement of plates, despite the small variation of the zero-phase frequency ( $10 \%$ in the operating range) that results in a small phase change, the effect of the diaphragm is very limited. It is then possible to hide the diaphragm in the metamaterial in this frequency range even in the presence of losses.

\section{B. Hiding zone}

Moreover, the stretch of the effective wavelength in the PAM should allow us to expand the hiding phenomenon to different locations of the scatterer. Figure 6 shows the evolution of the
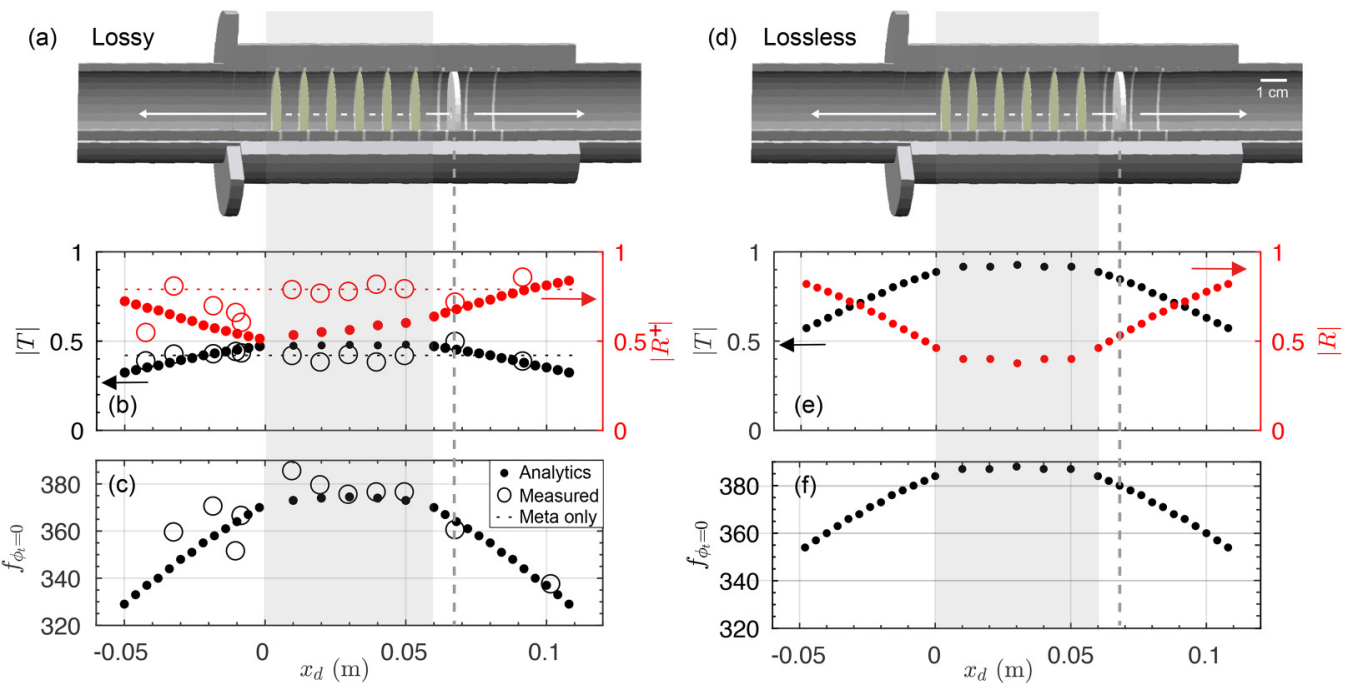

FIG. 6. Hiding zone of a $4 \mathrm{~mm}$ radius diaphragm in the PAM: with losses (a)-(c) and lossless (d)-(f). Sketches of the considered system (a) and (d), magnitude of the scattering parameters (transmission, black and left axis and reflection, red and right axis) at the zero-phase frequency of the system (b) and (e), and zero-phase frequency of the system (c) and (f). The circles, dots symbols, and dotted lines represent, respectively, the experimental, analytical data for the total system, and the experimental amplitude of the scattering parameters of the metamaterial alone at its zero-phase frequency. 
scattering amplitude and of the zero-phase frequency for different locations of the diaphragm along the metamaterial and in its vicinity. The two subplots Figs. 6(b) and 6(e) represent, in the lossy and lossless cases, respectively, the transmission magnitude (in black) and the reflection magnitude (in red) of the whole system at the zero-phase frequency $f_{\phi_{t}=0}$ for each location $x_{d}$ of the diaphragm.

As for the cloaking configuration, the presence of a noncentered obstacle causes an asymmetry in the system. It is then necessary to distinguish the reflection coefficients of the waves incident from each side of the device. The reflection coefficient $R^{+}$refers to the incidence from the left, while $R^{-}$refers to the incidence from the right. Since only one obstacle is considered here, the reflection $R^{+}$ generated by an obstacle placed between the 5th and 6th plates for a wave incident from the left is equivalent to the reflection $R^{-}$generated by an obstacle placed between the 1st and 2nd plates for a wave incident from the right. In the following, only the coefficient $R^{+}$will thus be presented. In addition, it is worth noting here that the system preserves its reciprocity condition but not the symmetry.

In the lossless case, no matter where the $4 \mathrm{~mm}$ radius diaphragm is along the metamaterial, the magnitudes of transmission and reflection coefficients remain constant and equal to the ones of the metamaterial alone. The hiding phenomenon covers the entire structure, which acts as a homogeneous and almost symmetrical material $\left(R^{+} \approx R^{-} \approx R\right)$. As soon as the viscothermal and viscoelastic losses are accounted for, the asymmetry of the system becomes much more visible, $R^{+} \neq R^{-}$, which results in a slight change of the reflection with the location of the diaphragm as we can see in Fig. 6(b). The agreement between the experimental results, represented by the circle symbols, and the analytical predictions, represented by the solid points, is very good on the transmission coefficient and the zero-phase frequency. More significant differences are, however, noticeable on the reflection, already noted in the case of the metamaterial alone [see Fig. 4(b)]. However, we find that regardless of the location of the diaphragm, the measured reflection and transmission of the whole system equal the ones of the PAM without diaphragm, the measured values of which are reminded by the dotted horizontal lines in Fig. 6(b).

According to these results, it is, therefore, possible to define a hiding zone corresponding to the whole metamaterial. The diaphragm can be dissimulated when placed between any plates, as long as the losses and impedance of the object to hide are controlled.

\section{CONCLUSIONS}

In this work, both the cloaking and hiding efficiency of a platetype metamaterial device have been investigated, using the DNZ regime associated with such systems. The study has been restricted to the case of a thin rigid diaphragm to be hidden due to the ease of manufacturing but can, nevertheless, be extended to any other obstacle.

We have shown that acoustic doping can be used to attain full cloaking in the lossless case, i.e., canceling any scattering from the diaphragm (total transmission, zero reflection, and zero-phase), by using either the impedance of the element to be concealed or an external impurity such as a designed Helmholtz resonator. We have reported that these two strategies have transformed the DNZ regime into DCNZ, thus fulfilling the requirement for cloaking. However, the effectiveness is significantly altered when the full viscothermal and viscoelastic losses are turned on. In addition, the length of the DNZ medium required, for the apparent phase to be equal with and without the cloaking device, results in a system size that is no longer subwavelength. In the self-doped configuration, the diaphragm aperture necessary to have a strong enough impedance to dope the system is so small that the viscothermal losses in the orifice make the diaphragm opaque. When doping is accomplished using a Helmholtz resonator impurity, the viscoelastic losses have been shown to avoid impedance matching, thus preventing full transmission and cloaking.

Accounting for the difficulties of obtaining cloaking condition using a device based on a $1 \mathrm{D}$ realistic PAM, we have shown that instead of using the PAM to suppress any scattering of the external sound field, the effective wavelength stretch produced by the DNZ regime of the PAM can be used to hide an obstacle inside. In doing so, the acoustic behavior of a medium filled with DNZ material (here the PAM) remains unchanged, regardless of whether an obstacle is present inside or not, as soon as its impedance is controled. Furthermore, we have shown that the long effective wavelength allows us to change the position of the obstacle inside the metamaterials without affecting the effectiveness of the hiding at all in the lossless case and with only a slight impact on the reflection in the lossy case.

We were thus able to report analytically, numerically, and experimentally on the presence of a hiding zone along the entire PAM. However, it is important to keep in mind that, as with most of the concealment strategies considered so far, the system proposed in this study is indeed efficient at a single frequency. Although limited by this narrow frequency band, the promising hiding strategy proposed in this paper alleviates the constraint of cloaking (a unitary transmission being almost impossible to reproduce with realistic devices and a non-subwavelength treatment length being necessary to reproduce the required apparent phase). This new hiding strategy could, therefore, help in the design of new applications, based on acoustic metamaterials involving plates, membranes, or any other DNZ system, capable of ensuring constant transmission even in the presence of obstacles.

\section{ACKNOWLEDGMENTS}

This article is based upon the work from COST Action DENORMS (No. CA15125), supported by COST (European Cooperation). The authors would like to thank the support of the ANR-RGC METARoom (No. ANR-18-CE08-0021) project. J. Christensen acknowledges the support from the European Research Council (ERC) through the Starting Grant No. 714577 PHONOMETA and from the MINECO through a Ramón y Cajal Grant (No. RYC-2015-17156).

\section{DATA AVAILABILITY}

The data that support the findings of this study are available from the corresponding author upon reasonable request.

\section{REFERENCES}

${ }^{1}$ A. N. Norris, “Acoustic cloaking," Acoust. Today 11, 38-46 (2015).

${ }^{2}$ R. Fleury, F. Monticone, and A. Alú, "Invisibility and cloaking: Origins, present, and future perspectives,” Phys. Rev. Appl. 4, 037001 (2015). 
${ }^{3}$ F. Zangeneh-Nejad and R. Fleury, "Active times for acoustic metamaterials," Rev. Phys. 4, 100031 (2019).

${ }^{4}$ F. Yang and Z. Lei Mei, "Parity-time symmetric cloak with isotropic modulation," J. Phys. D: Appl. Phys. 49, 21 LT01 (2016).

${ }^{5}$ H.-X. Li, M. Rosendo-López, Y.-F. Zhu, X.-D. Fan, D. Torrent, B. Liang, J.-C. Cheng, and J. Christensen, "Ultrathin acoustic parity-time symmetric metasurface cloak," Research 2019, 1-7 (2019).

${ }^{6} \mathrm{H}$. Chen and C. T. Chan, "Acoustic cloaking in three dimensions using acoustic metamaterials,” Appl. Phys. Lett. 91, 183518 (2007).

7. B. Pendry and J. Li, "An acoustic metafluid: Realizing a broadband acoustic cloak,” New J. Phys. 10, 115032 (2008).

${ }^{8}$ A. N. Norris, "Acoustic cloaking theory," Proc. R. Soc. A 464, 2411-2434 (2008).

${ }^{9}$ S. A. Cummer and D. Schurig, "One path to acoustic cloaking," New J. Phys. 9 , $45-45$ (2007).

${ }^{10} \mathrm{H}$. Chen and C. T. Chan, "Acoustic cloaking and transformation acoustics," J. Phys. D: Appl. Phys. 43, 113001 (2010).

${ }^{11}$ J. B. Pendry, "Controlling electromagnetic fields," Science 312, 1780-1782 (2006).

${ }^{12}$ D. Schurig, J. B. Pendry, and D. R. Smith, "Calculation of material properties and ray tracing in transformation media," Opt. Express 14, 9794 (2006).

${ }^{13}$ S. A. Cummer, J. Christensen, and A. Alú, "Controlling sound with acoustic metamaterials," Nat Rev Mater 1, 16001 (2016).

${ }^{14}$ S. A. Cummer, B.-I. Popa, D. Schurig, D. R. Smith, J. Pendry, M. Rahm, and A. Starr, "Scattering theory derivation of a 3D acoustic cloaking shell," Phys. Rev. Lett. 100, 024301 (2008).

${ }^{15} \mathrm{D}$. Torrent and J. Sánchez-Dehesa, "Acoustic cloaking in two dimensions: A feasible approach,” New J. Phys. 10, 063015 (2008).

${ }^{16}$ B.-I. Popa and S. A. Cummer, "Design and characterization of broadband acoustic composite metamaterials," Phys. Rev. B 80, 174303 (2009).

${ }^{17}$ L. Zigoneanu, B.-I. Popa, A. F. Starr, and S. A. Cummer, "Design and measurements of a broadband two-dimensional acoustic metamaterial with anisotropic effective mass density," J. Appl. Phys. 109, 054906 (2011).

${ }^{18}$ G. W. Milton and A. V. Cherkaev, "Which elasticity tensors are realizable?," J. Eng. Mater. Technol. 117, 483-493 (1995).

${ }^{19}$ C. L. Scandrett, J. E. Boisvert, and T. R. Howarth, "Acoustic cloaking using layered pentamode materials,” J. Acoust. Soc. Am. 127, 2856-2864 (2010).

${ }^{20}$ N. H. Gokhale, J. L. Cipolla, and A. N. Norris, "Special transformations for pentamode acoustic cloaking,” J. Acoust. Soc. Am. 132, 2932-2941 (2012).

${ }^{\mathbf{2 1}}$ Y. Chen, X. Liu, and G. Hu, "Latticed pentamode acoustic cloak," Sci. Rep. 5, 15745 (2015)

${ }^{22}$ V. M. García-Chocano, L. Sanchis, A. Díaz-Rubio, J. Martínez-Pastor, F. Cervera, R. Llopis-Pontiveros, and J. Sánchez-Dehesa, "Acoustic cloak for airborne sound by inverse design," Appl. Phys. Lett. 99, 074102 (2011).

${ }^{23}$ L. Sanchis, V. M. García-Chocano, R. Llopis-Pontiveros, A. Climente, J. Martínez-Pastor, F. Cervera, and J. Sánchez-Dehesa, "Three-dimensional axisymmetric cloak based on the cancellation of acoustic scattering from a sphere," Phys. Rev. Lett. 110, 124301 (2013).

${ }^{24}$ B.-I. Popa, L. Zigoneanu, and S. A. Cummer, "Experimental acoustic ground cloak in air," Phys. Rev. Lett. 106, 253901 (2011).

${ }^{25}$ L. Zigoneanu, B.-I. Popa, and S. A. Cummer, "Three-dimensional broadband omnidirectional acoustic ground cloak," Nat. Mater 13, 352-355 (2014).

${ }^{\mathbf{2 6}} \mathrm{Y}$. Jin, X. Fang, Y. Li, and D. Torrent, "Engineered diffraction gratings for acoustic cloaking," Phys. Rev. Appl. 11, 011004 (2019).
${ }^{27}$ Y. Yang, H. Wang, F. Yu, Z. Xu, and H. Chen, "A metasurface carpet cloak for electromagnetic, acoustic and water waves,” Sci. Rep. 6, 20219 (2016).

${ }^{28}$ C. Faure, O. Richoux, S. Félix, and V. Pagneux, "Experiments on metasurface carpet cloaking for audible acoustics," Appl. Phys. Lett. 108, 064103 (2016).

${ }^{29} \mathrm{M}$. Dubois, C. Shi, Y. Wang, and X. Zhang, "A thin and conformal metasurface for illusion acoustics of rapidly changing profiles," Appl. Phys. Lett. 110, 151902 (2017)

${ }^{30}$ S. Zhai, H. Chen, C. Ding, L. Li, F. Shen, C. Luo, and X. Zhao, "Ultrathin skin cloaks with metasurfaces for audible sound," J. Phys. D: Appl. Phys. 49, 225302 (2016).

${ }^{31}$ H. Esfahlani, S. Karkar, H. Lissek, and J. R. Mosig, "Acoustic carpet cloak based on an ultrathin metasurface," Phys. Rev. B 94, 014302 (2016).

${ }^{32}$ L.-Y. Zheng, Y. Wu, X. Ni, M.-H. Chen, Z.-G. Lu, and Y.-F. Chen, "Acoustic cloaking by a near-zero-index phononic crystal,” Appl. Phys. Lett. 104, 161904 (2014).

${ }^{33}$ J. Zhao, Z. N. Chen, B. Li, and C.-W. Qiu, "Acoustic cloaking by extraordinary sound transmission,” J. Appl. Phys. 117, 214507 (2015).

${ }^{34} \mathrm{R}$. Fleury and A. Alú, "Extraordinary sound transmission through density-near-zero ultranarrow channels," Phys. Rev. Lett. 111, 055501 (2013).

${ }^{35}$ R. Graciá-Salgado, V. M. García-Chocano, D. Torrent, and J. Sánchez-Dehesa,

"Negative mass density and $\rho$-near-zero quasi-two-dimensional metamaterials: Design and applications," Phys. Rev. B 88, 1-12 (2013).

${ }^{36}$ Z. Yang, J. Mei, M. Yang, N. H. Chan, and P. Sheng, "Membrane-type acoustic metamaterial with negative dynamic mass," Phys. Rev. Lett. 101, 1-4 (2008).

${ }^{37} \mathrm{~F}$. Bongard, H. Lissek, and J. R. Mosig, "Acoustic transmission line metamaterial with negative/zero/positive refractive index," Phys. Rev. B 82, $094306(2010)$

${ }^{38}$ J. J. Park, K. J. Lee, O. B. Wright, M. K. Jung, and S. H. Lee, "Giant acoustic concentration by extraordinary transmission in zero-mass metamaterials," Phys. Rev. Lett. 110, 1-5 (2013).

${ }^{39} \mathrm{M}$. Malléjac, A. Merkel, J. Sánchez-Dehesa, J. Christensen, V. Tournat, J. P. Groby, and V. Romero-García, "Zero-phase propagation in realistic platetype acoustic metamaterials," Appl. Phys. Lett. 115, 134101 (2019).

${ }^{40}$ Y. Gu, Y. Cheng, J. Wang, and X. Liu, "Controlling sound transmission with density-near-zero acoustic membrane network," J. Appl. Phys. 118, 024505 (2015).

${ }^{41}$ M. Malléjac, A. Merkel, V. Tournat, J.-P. Groby, and V. Romero-García, "Doping of a plate-type acoustic metamaterial,” Phys. Rev. B 102, 060302(R) (2020).

${ }^{42}$ V. C. Henríquez, V. M. García-Chocano, and J. Sánchez-Dehesa, "Viscothermal losses in double-negative acoustic metamaterials," Phys. Rev. Appl. 8, 014029 (2017). ${ }^{43} \mathrm{~B}$. H. Song and J. S. Bolton, "A transfer-matrix approach for estimating the characteristic impedance and wave numbers of limp and rigid porous materials," J. Acoust. Soc. Am. 107, 1131-1152 (2000).

${ }^{44} \mathrm{M}$. R. Stinson, "The propagation of plane sound waves in narrow and wide circular tubes, and generalization to uniform tubes of arbitrary cross-sectional shape," J. Acoust. Soc. Am. 89, 550-558 (1991).

${ }^{45}$ I. Liberal, A. M. Mahmoud, Y. Li, B. Edwards, and N. Engheta, "Photonic doping of epsilon-near-zero media," Science 355, 1058-1062 (2017).

${ }^{46}$ I. Liberal, Y. Li, and N. Engheta, "Reconfigurable epsilon-near-zero metasurfaces via photonic doping," Nanophotonics 7, 1117-1127 (2018).

${ }^{47} \mathrm{~V}$. C. Henríquez, V. M. García-Chocano, and J. Sánchez-Dehesa, "Viscothermal losses in double negative acoustic metamaterials," Phys. Rev. Appl. 8, 014029 (2017). 